\title{
Multi-Step Lining-up Correction of the CLIC Trajectory
}

\author{
E. T. d'Amico, G. Guignard, CERN, 1211 Geneva 23, Switzerland
}

\begin{abstract}
In the CLIC main linac it is very important to minimise the trajectory excursion and consequently the emittance dilution in order to obtain the required luminosity. Several algorithms have been proposed and lately the ballistic method has proved to be very effective. The trajectory correction method described hereafter retains the main advantages of the latter while adding some interesting features. It is based on the separation of the unknown variables like the quadrupole misalignments, the offset and slope of the injection straight line and the misalignments of the beam position monitors (BPM). This is achieved by referring the trajectory relatively to the injection line and not to the average pre-alignment line and by using two trajectories each corresponding to slightly different quadrupole strengths. A reference straight line is then derived onto which the beam is bent by a kick obtained by moving the first quadrupole. The other quadrupoles are then aligned on that line. The quality of the correction depends mainly on the BPM's and micro-movers' resolution and on the stability of the quadrupole strengths. Simulation statistics show that the beam offset from the center of the quadrupoles is typically $1.5 \mu \mathrm{m}$ r.m.s.
\end{abstract}

\section{INTRODUCTION}

Two studies of trajectory correction are underway for CLIC, the ballistic alignment method [1] [2] and the Multistep Lining-up (ML) described in this paper [3].

The latter has in common with the NLC correction and the ballistic method the idea to align as well as possible the main components of the linac on a straight line defined by the beam. The development of ML is also based on the observation that to align the quadrupoles on a reference line is more important that the real choice and straightness of this line, provided it is not too far away from the averagealignment line fixed by the positions of the components resulting from the pre-alignment. Instead of switching-off the quadrupoles, the ML method relies on a small change of their strengths in a linac section and on measurements of trajectory differences as in the dispersion-free correction [4] although used otherwise. This has the advantages of minimizing the heat-load variations, making the remanentfield and hysteresis effects negligible, keeping the beam focused and not too distant from the center of the elements (reducing wakefield effects) and eventually allowing on-line corrections by matching the detuned section to the rest of the linac. Trajectories are referred to the section injection line in order to make their differences independent of the injection parameters. As a first step, these differences are used in ML to estimate the off-sets of the quadrupoles relative to the same line. In the second step, a least square fit of the BPM measurements allows a good estimation of the injection parameters and the definition of a reference line onto which the quadrupoles are actually moved. The beam is bent towards this line by moving the first quadrupole of the section. As a last step in the correction, the BPMs sitting at the head of each girder are also displaced toward the reference line (by nullifying their measurements). This also aligns all the girders, reducing the cavity misalignments to their scattering with respect to each girder. Because the optics model is perturbed by the wakefields and other imperfections, the correction is an iterative process that rapidly converges [5]. Investigations show that with acceptable tolerances on the measurementand micromover-resolution, acquisition noise and precision of the quadrupole power supplies, the ML trajectory correction allows a very good control of the beam offsets with respect to the center of the quadrupoles. Numerical investigations of the emittance blow-up after the ML correction will be done as soon as the algorithm is entirely implemented in a tracking program.

\section{DESCRIPTION OF THE METHOD}

The magnetic quadrupoles and the beam position monitors (BPM) of the CLIC main linac are assumed to be prealigned around an ideal line referred to as the average prealignment line. The optics is a FODO with a focal length $f$ and a distance between quadrupoles $L$. The BPM is placed at a distance $l$ in front of the quadrupole. Considering a section of $N$ quadrupoles, let $\delta_{q, i}$ and $\delta_{p, i}$ be the offsets of the $\mathrm{i}$-th quadrupole and i-th BPM from the average prealignment line respectively. The beam entering the first quadrupole follows the straight line defined by the injection offset $x_{i n j}$ and slope $s_{i n j}$ (see Fig.1). The beam trajectory

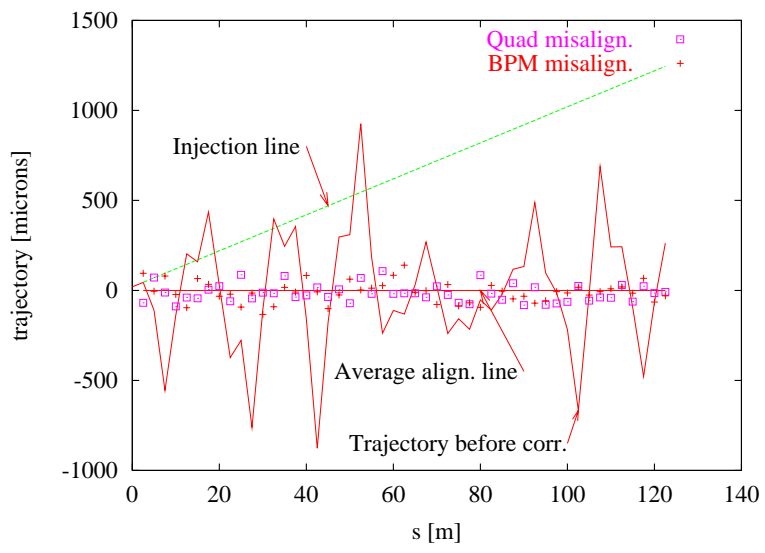

Figure 1: Beam trajectory and injection line with quadrupole and BPM offsets 
ottset trom the 1-th BPIM center is given by the following expression [3] :

$$
\begin{aligned}
t_{i}= & x_{i n j}\left(1-\sum_{k=1}^{i-1} u_{i, k}\right)+s_{i n j}\left(i L-l-L \sum_{k=1}^{i-1} u_{i, k} k\right) \\
& +\sum_{k=1}^{i-1} u_{i, k} \delta_{q, k}-\delta_{p, i}
\end{aligned}
$$

where $u_{i, k}=L \sum_{j=k}^{i-1} p_{j, k}-l p_{i-1, k}$ the $p_{j, k}$ being obtained iteratively [3]. The coefficients $u_{i, k}$ describe the beam transport in the FODO channel at each BPM and only depend on the lattice parameters $L, l, f$. The BPM unknown misalignments can be canceled out by making the differences of two trajectories at two different quadrupole strengths as in the dispersion-free correction [4]. The determinant of the resulting underdetermined linear system is null because the coefficients of $x_{i n j}$ and $s_{i n j}$ become linear combinations of the coefficients of the $\delta_{q, k}$. This fact suggests that it is possible to make the coefficients of the unknown variables $x_{i n j}$ and $s_{i n j}$ of the expression (1) independent of the quadrupole strengths by changing the reference system of the quadrupole offsets. The obvious choice is the injection line itself. The result obtained from (1) is:

$$
t_{i}=x_{i n j}+s_{i n j}(i L-l)+\sum_{k=1}^{i-1} u_{i, k} d_{q, k}-\delta_{p, i}
$$

where $d_{q, k}=\delta_{q, k}-x_{i n j}-s_{i n j} k L$. To get rid of both the BPM offsets $\delta_{p, i}$ and the injection parameters another beam trajectory is generated by slightly increasing the quadrupole focal length (typically $5 \%$ ). The difference between the two trajectories inside each BPM, one at the nominal focal length $f_{1}$ and one at the focal length $f_{2}$, is

$$
t_{i}\left(f_{1}\right)-t_{i}\left(f_{2}\right)=\sum_{k=1}^{i-1}\left[u_{i, k}\left(f_{1}\right)-u_{i, k}\left(f_{2}\right)\right] d_{q, k}
$$

from which the estimated quadrupole offsets $\hat{d}_{q, k}$ relative to the injection line are deduced. The estimation errors $\varepsilon_{d, k}=\hat{d}_{q, k}-d_{q, k}$ depend on the BPM resolutions and on the precision of the quadrupole strengths. Subtracting the estimated contribution of the quadrupole offsets from expression (2) a virtual trajectory around the injection line can be introduced:

$$
\begin{aligned}
t_{v, i}\left(f_{1}\right) & =t_{i}\left(f_{1}\right)-\sum_{k=1}^{i-1} u_{i, k}\left(f_{1}\right) \hat{d}_{q, k} \\
& =x_{i n j}+s_{i n j}(i L-l)-\delta_{p, i}-\sum_{k=1}^{i-1} u_{i, k}\left(f_{1}\right) \varepsilon_{d, k} .
\end{aligned}
$$

Observing that the BPM offsets and the estimation errors $\varepsilon_{d, k}$ are randomly distributed, the injection parameters can be estimated by the Least Squares Method. The reference straight line on which the beam should be directed is the straight line which passes inside the hrst quadrupole at the estimated offset $\hat{x}_{i n j}+\hat{s}_{i n j} L$ from the average pre-alignment line and intersects this one inside the last quadrupole of the section. The beam is bent in the direction of the reference line by moving the first quadrupole of the section by $\Delta_{q, 1}=-\hat{d}_{q, 1}+f \Delta s$ where $\Delta s$ is the difference between the reference and estimated injection slope. The other quadrupoles should be aligned onto the reference line by displacing them by the quantities $\Delta_{q, i}=-\hat{d}_{q, i}+\Delta s(i-1) L$. The broken line followed by the beam after these movements is different from the reference line as it is shown in Fig.2. However it has been established [3] that the offset $\delta_{t, i}$ of the beam relative to the $\mathrm{i}$-th quadrupole center is given by

$$
\delta_{t, i}=\varepsilon_{d, i}-\sum_{k=1}^{i-1} u_{i, k}^{\prime} \varepsilon_{d, k}
$$

where the $u_{i, k}^{\prime}$ describe the beam transport at each quadrupole and are equal to the $u_{i, k}$ for $l=0$. It is noteworthy that the above-mentioned offsets do not depend on the reference line parameters.

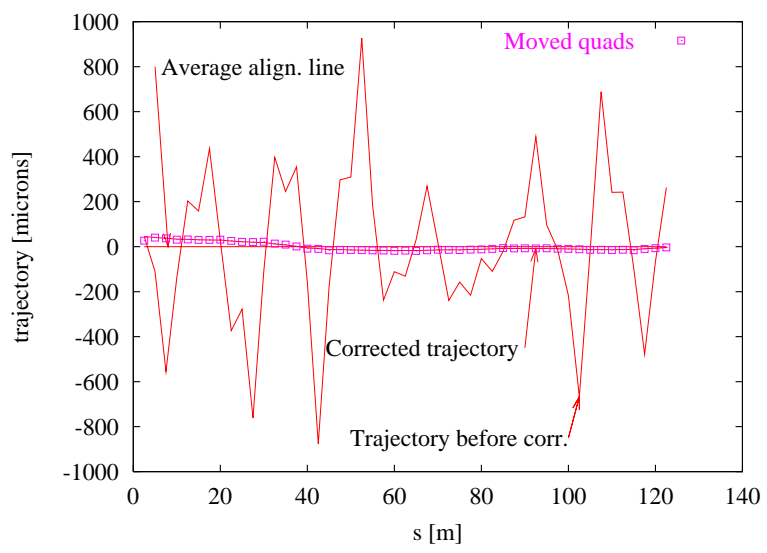

Figure 2: Beam trajectory before and after ML correction

\section{CORRECTION STRATEGY}

In this section, a correction procedure based on the Multistep Lining-up method is proposed. As the result of the survey all the components of the linac are assumed to be randomly scattered around the so-called averagedprealignment line. Acceptable r.m.s. offset amplitudes are of the order of $50 \mu \mathrm{m}$ r.m.s. for both the quadrupoles and BPM. For the accelerating structures (cavities) placed on a single girder, the relevant quantity is their pre-alignment offset with respect to the BPM sitting on the same girder. This has to be within 2 and $10 \mu m$ r.m.s. . The relative misalignments between girders are given by those of the BPMs. For the first correction a single bunch is injected. Sections of $N$ quadrupoles are successively dealt with in the linac. $N$ is optimized for an accurate definition of the reference line and a good least squares fit of the measure- 
ments over the BPMs used to estımate the injection parameters. Preliminary modeling shows that $N$ can be as large as 50 or more, which opens the way to a correction section which could in principle be as long as a linac sector, defined by the FODO lattice being constant. The beam is injected into the nominal lattice and the beam positions are measured at each BPM. To gain a factor 10 on the resolution and acquisition errors, measurements should be averaged over typically 100 pulses (about $1 s$ ). The beam is then injected into a lattice only detuned in the section considered and betatron-matched to the rest of the linac. The focal distance increment by $\sim 5 \%$ has been found to be sufficient because the difference between the two trajectories is enhanced by the phase advance shift. The beam positions are again measured and averaged over 100 pulses.

The ML algorithm is now applied following these steps:

1. Isolate the contribution of the quadrupole misalignments by building up the differences between the two trajectories. Solve the obtained triangular system of $N-1$ equations and $N-1$ unknowns which are the quadrupole displacements $d_{q, k}$ with respect to the injection line. Actually the results will be estimations $\hat{d}_{q, k}$. Restore the nominal lattice and suppress the betatron-matching.

2. Subtract the estimated contribution of the quadrupole misalignments from the nominal trajectory BPM readings. Assuming that the BPM misalignments are randomly distributed, these measurements scatter around the injection straight line. Estimates of its offset and slope are obtained by a least squares fit.

3. Compute the change of the slope (kick) that would steer the beam towards the average-prealignment line by using the estimated injection parameters. The obtained straight line is the reference line on which the quadrupoles should be aligned. To achieve this, the first quadrupole of the bin should be displaced in order to apply the computed kick. All the other quadrupoles should then be moved in order to align them onto the reference line.

4. Move all the BPMs sitting at the head of each girder to the reference line, by nullifying their reading within their resolution. This is a kind of "calibration" of the measurement system.

Moving the BPMs will also realign the girders. The cavity position scattering is consequently reduced to their starting pre-alignment imperfections on a single girder. The wake-field effects are accordingly decreased limiting the emittance growth. At least one iteration of this process is necessary because the lattice model used in the algorithm does not include the wake-fields. The procedure described here must be repeated section after section over the whole linac, before the full-intensity beam can be injected.

Simulations assume a BPM resolution of $0.1 \mu \mathrm{m}$, an acquisition noise of $0.1 \mu \mathrm{m}$, a micro-mover resolution of
U.J $\mu \mathrm{m}$ and a precision of the quadrupole strength of $\Delta f / f=5 \times 10^{-4}$. Results indicate that the reference line deviation with respect to the average prealignment line is of the order of $30 \mu \mathrm{m}$ r.m.s. at the end of a section of $125 \mathrm{~m}$. The most important result is that, although the actual line followed by the beam does not exactly coincide with the reference line, the remaining offsets between the beam and the quadrupole-centers (independent of the initial pre-alignment and of the line parameters) is of the order of $\sim 1.5 \mu \mathrm{m}$ r.m.s. (see Fig.2).

Turning to time-dependent drifts of the components after the first correction has been completed, the BPMs may begin to measure non-zero deviations if the beam does not follow the change of the geometry. An on-line, one-to-one feedback can be applied in the case of smooth displacements as in an ATL-model. When BPM measurements indicate short-range position variations or the beam moving away from the linac component centers, the ML correction has to be repeated.

Considering now the first linac-section, the reference line obtained by ML can be used to correct for the injection jitter while maintaining the same trajectory over the rest of the linac. For this, it is necessary to apply two correction kicks, near the first quadrupole and for instance near one in the middle of the section, in order to maintain the beam on a constant trajectory through the BPMs following the second quadrupole (by keeping their reading equal to zero). To use this correction as a feedback may require two fast kickers near the two quadrupoles mentioned while static corrections of the injection can be done by actually moving the two quadrupoles.

Effects of jitter during BPM acquisitions and of the terrestrial magnetic field remain to be taken into account [6]. Full simulations with tracking programs [7] should be carried out to check the robustness of the method. The hope is to apply the ML correction on-line with only small focal changes and sector-matching, without interrupting the beam acceleration.

\section{REFERENCES}

[1] T. O. Raubenheimer, D. Schulte,'The ballistic alignment method", this conference.

[2] D. Schulte,'The CLIC Main Linac Lattice at $1 \mathrm{TeV}$ ', CLIC Note 356, 1998.

[3] E. T. d'Amico and G. Guignard,"A trajectory correction based on Multi-step Lining-up for the CLIC main Linac", CLIC Note 388, 1999.

[4] T. O. Raubenheimer,'The Generation and Acceleration of Low Emittance Flat Beams for Future Linear Colliders", SLAC Report 387, 1991.

[5] The NLC Design Group,'Zeroth-Order Design Report for the Next Linear Collider", SLAC report 474, 1996.

[6] S. Fartoukh, D. Schulte, Private communications.

[7] G. Guignard and J. Hagel,'MUSTAFA- A Tool for Numerical Simulations of the Beam Behaviour in a Linac", Proc. of the LINAC98 conf., Chicago, 1998. 